\title{
TRADUCCIÓN ECONÓMICA, FINANCIERA Y COMERCIAL: APROXIMACIÓN A ASPECTOS TEÓRICOS. ESTUDIO BASADO EN ENCUESTAS
}

Daniel Gallego Hernández

daniel.gallego@ua.es Universidad de Alicante

Geoffrey S. Koby

gkoby@kent.edu

Kent State University

Verónica Román Mínguez

veronica.roman@uam.es Universidad Autónoma de Madrid

\section{Resumen}

Este artículo pretende dar respuesta a una serie de preguntas que tanto los formadores de traductores en el ámbito de la economía y los negocios como los investigadores en materia de traducción en tales ámbitos suelen plantearse. En este sentido, abordamos el tema de las denominaciones que suele recibir esta práctica traductora por medio de una encuesta destinada a especialistas y formadores de traductores en el ámbito universitario, centrada en diferentes materias o áreas del mundo de la economía, el comercio y las finanzas. Asimismo, utilizamos esta encuesta para plantearnos las líneas de investigación en las que quizá cabría hacer más hincapié y reflexionar sobre el término teoría en la formación en esta práctica de la traducción.

\begin{abstract}
"Economic, financial, and commercial translation: an approach to theoretical aspects. A survey-based study"

This article seeks to answer a number of questions often raised by both translator trainers and translation researchers in the field of economics and business. The subject areas often attributed to this field of translation practice are examined using a
\end{abstract}


survey administered to professionals and university-level translator trainers, focusing on a range of subjects and areas in the world of economics, commerce, and finance. We also use this survey to examine lines of research where more emphasis may be appropriate, as well as how the term theory factors into training for the practice of translation.

Palabras clave: Traducción económica. Traducción comercial. Traducción financiera. Teoría. Encuesta.

Keywords: Economic translation. Commercial translation. Financial translation. Theory. Survey.

Editorial article, received on September 19, 2015. 


\section{Introducción: etiquetas de traducción en el ámbito de la economía, el comercio y las finanzas}

Son distintas las etiquetas que aluden a la práctica de la traducción en el ámbito de la economía y los negocios. Por una parte, encontramos las denominaciones de traducción económica o de textos económicos, que pueden entenderse de manera laxa. Por ejemplo, el trabajo de Bocquet (1993) surge como reflexión tras unos encuentros entre traductores profesionales del sector financiero y bancario. Por su parte, Svendsen (2001: 40) se refiere a la denominación economic translation centrándose, en esencia, "on some aspect of either business, the financial sector or macro-economics". Gallego (2012, 2014) utiliza esta denominación para englobar todo tipo de traducción especializada en tales ámbitos. Por su parte, Román (2015) habla de traducción en el ámbito de la economía y los negocios. Ahora bien, otros autores aluden a la traducción económica como una práctica algo más restringida. Es el caso de Llombart (2009), cuyo repertorio de traducciones tiene que ver con obras teóricas sobre economía y artículos especializados.

A la denominación de traducción económica se suma la de traducción comercial. Guével (1990: 154), por ejemplo, alude a la traduction commerciale o traduction dans le domaine des affaires centrándose en el término affaires, concebido como "un champ d'application de l'économie, du point de vue de l'entreprise. Ce domaine regroupe les multiples activités économiques de l'entreprise, en particulier celles qui ont trait au commerce, aux finances et à la gestion". Gouadec (2007: 11) define la traducción económica como "translation of documents relating to the economy", lo que aparentemente incluye la traducción comercial, pues no la menciona en su listado de campos de traducción. En una línea de pensamiento similar se manifiesta Guidère (2008: 14), quien concibe la traduction commerciale como aquella "commanditée par une entreprise" y relacionada especialmente con los sitios web comerciales y la promoción de productos y servicios en los mercados internacionales. También en este orden de ideas, Lahlali \& Hatab (2014: 55) tienen una visión muy amplia de la traducción económica, "required for marketing and com-

merce, banking and the investment sector, the insurance industry, accounting 
and auditing, and marketing research", que incluye, por tanto, no solo la traducción comercial, sino también la traducción financiera. Lie (1995: 95), en cambio, considera que la etiqueta commercial translation, "defined simply, is any translation work undertaken and required directly or indirectly for any commercial purpose [...] the label 'commercial translator' can be inclusively applied to anyone engaged in translation work relating (even remotely) to any of these activities". Por su parte, para Mayoral (2007: 33) la traducción comercial tiene que ver con las operaciones comerciales y, por tanto, con un grupo restringido de documentos que comparten formas y funciones muy similares (documentos de pago, avales, documentos de transporte, facturas, etc.). Por último, la entrada commercial translation de la enciclopedia de Olohan (2009: 40-41) señala lo siguiente:

Commercial translation, financial translation, economic translation, business translation and other, similar terms do not correspond so readily to existing classifications of knowledge. There is thus no consensus on how to label or define this translation activity. The term used here [commercial translation] is one of convenience, intended to cover the translation of all texts used in business contexts, excluded technical and legal texts.

También se utiliza la denominación de traducción financiera para aludir a otros campos. Según Durban (2005: 63-65) este tipo de traducción tiene lugar en la comunicación financiera, los análisis financieros y la macroeconomía, el análisis financiero, los estados financieros, y las operaciones financieras. Rochard (2005: 7), por su parte, sostiene que la traduction financière es "une appellation fourre-tout qui va de l'économie politique au financement du développement en passant par la Bourse, les assurances, la comptabilité", y que se inserta en un campo que "recouvre de multiples segments et dans lequel les intervenants doivent sans cesse s'adapter à des tendances et des logiques très différentes". Esta visión general es compartida por Gouadec (2007: 11), quien la define como "translation of documents related to banking and finance".

De esta en absoluto exhaustiva reseña de trabajos que, de una manera u otra, aluden a la práctica de la traducción para el ámbito de la economía y los negocios, puede inferirse la dificultad a la que se refieren algunos autores (Heras 2002: 50; Mayoral 2007: 36) al tratar de definir o concebir la práctica de la traducción económica.

Ante este panorama, pretendemos aportar nuestro pequeño grano de arena a la hora de concebir este tipo de denominaciones y tratar de facilitar, de este modo, tanto la formación de este ámbito de la traducción como su investigación. Para ello, diseñamos un estudio basado en encuestas para conocer qué áreas de especialización pueden atribuirse a las etiquetas de economía, 
comercio y finanzas. Dicha encuesta está destinada no solo a especialistas profesionales y universitarios de tales ámbitos, sino también a formadores de traductores para estas áreas. Con ello, podremos contrastar los datos de especialistas y profesores y, en consecuencia, abordar, entre otros aspectos, la cuestión del conocimiento temático que el traductor debe adquirir para realizar traducciones en este ámbito de especialidad.

\section{Estudio basado en encuestas}

En este apartado presentamos el diseño de la encuesta, así como los resultados obtenidos de ambos grupos.

\subsection{Metodología}

Los datos presentados están extraídos de una encuesta diseñada con los siguientes objetivos:

- Conocer las materias que pueden enmarcarse en las etiquetas de economía, comercio y finanzas

- Conocer qué materias se trabajan en el aula de traducción económica en el ámbito universitario.

- Conocer en qué aspectos cabría hacer hincapié a la hora de investigar la traducción económica

Según el marco teórico de Visauta (1989: 262-264), se trata de una encuesta descriptiva con fines específicos (optimizar la formación e investigación en traducción), referida a hechos y opiniones (la formación de la traducción y la concepción de diferentes denominaciones), autorrellenada (distribuida a través de internet), y sincrónica (abierta desde principios de abril hasta final de junio de 2015).

Para ello, elaboramos dos cuestionarios bilingües (español e inglés): uno destinado a profesionales del mundo de la economía, el comercio y las finanzas, y otro, a formadores de traductores en tales ámbitos.

El diseño del primer cuestionario se distribuía en dos bloques: uno referido al perfil del profesional (edad, sexo, experiencia, nivel de estudios), y otro, a las materias que enmarcaría en uno u otro campo (se propuso un listado con diferentes materias ${ }^{1}$ y se formuló la siguiente pregunta: “¿En cuál

1. Las materias en cuestión fueron las siguientes: administración de empresas; análisis de riesgo; auditoría y consultoría; banca comercial; banca de inversión (MBO, OPA); bolsa; certificados de acciones; comercio; comercio exterior; contabilidad (cuentas anuales o 
de los campos situados en el encabezado (economía, comercio y finanzas) situaría cada materia?", los encuestados podían incluir las materias en más de un campo). ${ }^{2}$ Por su parte, el segundo cuestionario, además de estos dos bloques, contenía preguntas relacionadas con la investigación y enseñanza de la traducción:

- ¿Cuál es el número total de horas que dedica a la traducción económica, comercial y financiera en su programa?

- ¿En qué nivel enseña traducción económica, comercial o financiera?

- En el marco de la traducción económica, comercial o financiera, ¿qué es para usted la teoría? [Teoría de la traducción; Conocimientos del ámbito general de la economía, comercio y finanzas; Conocimientos del área económica concreta para la que se traduce; Otros]

- ¿En qué medida considera que la teoría es esencial en la práctica de la traducción para el ámbito económico, comercial y financiero?

- ¿Considera necesario impartir conocimientos económicos, comerciales y financieros en materias de traducción económica, comercial y financiera?

- ¿Qué aspectos considera que son más importantes a la hora de enseñar la traducción económica, comercial y financiera? [Terminológicos; Comunicativos; Textuales; Otros]

- iCree que es necesario ser un experto en economía, comercio o finanzas para traducir textos económicos, comerciales o financieros? ${ }^{3}$

estados financieros, IFRS, GAAP); contabilidad de gestión o directiva; contratos de alta dirección; contratos de compraventa de acciones; contratos del ámbito bancario; contratos financieros; contratos mercantiles (leasing, factoring, renting, contraltos de suministro, etc.); controlling; desarrollo de negocio; deuda pública; documentos financieros del ámbito internacional; documentos societarios (estatutos sociales, notificaciones y actas de reuniones del consejo de administración y de la junta general de accionistas, delegaciones de voto); estadística y estudios de mercado; financiación; finanzas corporativas; fiscalidad internacional; gobierno corporativo; información para accionistas e inversores (informes anuales, informes anuales de gobierno corporativo, informes de gestión); informes fiscales; instrumentos financieros (fondos de inversión, derivados); instrumentos negociables (cheques, letras de cambio, pagarés); logística; macroeconomía; marketing; mercado de trabajo; mercados financieros; microeconomía; negocio al por menor; operaciones corporativas ( $\mathrm{M} \& \mathrm{~A}, \mathrm{MBO}, \mathrm{IPO})$; planes de opciones sobre acciones; presupuestos, órdenes de compras, notas de abono; project finance; protocolo; recursos humanos (en el ámbito de negocios); seguros; socioeconomía.

2. En el caso del cuestionario destinado a formadores de traductores, se les preguntó igualmente si incluyen en sus clases alguna de estas materias.

3. Esta pregunta también se formuló al grupo de profesionales especialistas. 
- ¿En qué aspectos piensa que el investigador en traducción económica, comercial y financiera debería hacer más hincapié? [Cómo enseñar la traducción económica, comercial y financiera; Desarrollo de productos terminográficos; Estudios contrastivos de géneros textuales de la economía, el comercio y las finanzas; Estudios sociales de cuál es el perfil del traductor económico, comercial y financiero; Estudios cognitivos sobre el proceso de traducción económica, comercial y financiera; Otros]

La encuesta se lanzó fundamentalmente a un grupo cerrado de profesionales y docentes universitarios de programas de grado y máster, aunque también se distribuyó en la lista de la Asociación Americana de Estudios de Traducción e Interpretación (American Translation and Interpreting Studies Association, ATISA). No obstante, las respuestas recibidas de fuera de España fueron muy escasas, y ninguna de los Estados Unidos.

\subsection{Resultados}

\subsubsection{Descripción de encuestados}

Treinta y ocho (19 hombres y 19 mujeres) profesionales respondieron a la encuesta. La mayor parte de ellos ejerce en España (35) en el ámbito profesional, aunque algunos lo hacen también en el ámbito universitario:

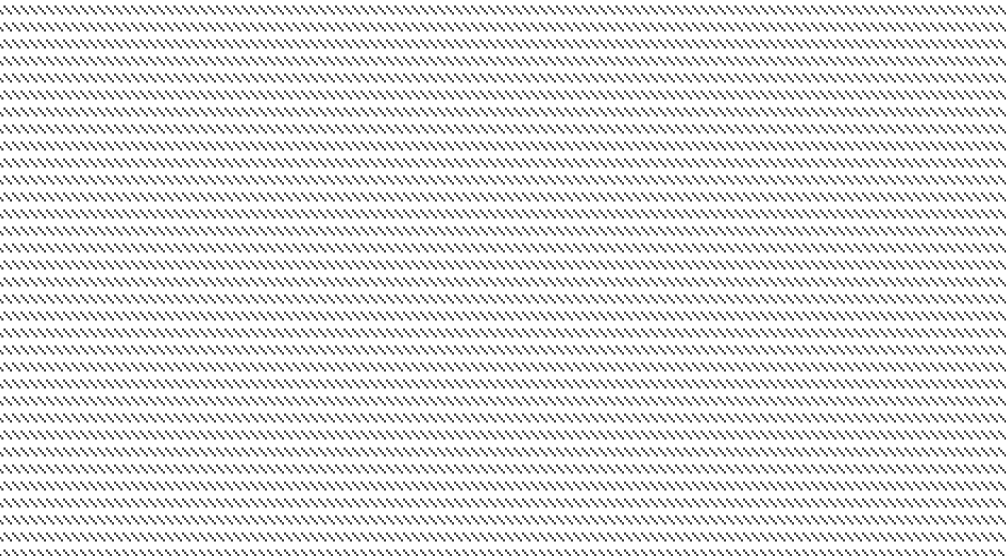

Gráfico 1: ¿En qué campo trabaja? 
Se trata de un conjunto de profesionales con más de 10 años de experiencia, en su mayor parte, licenciados (19) o con estudios de máster (17). El gráfico 2 muestra su distribución por años de experiencia:

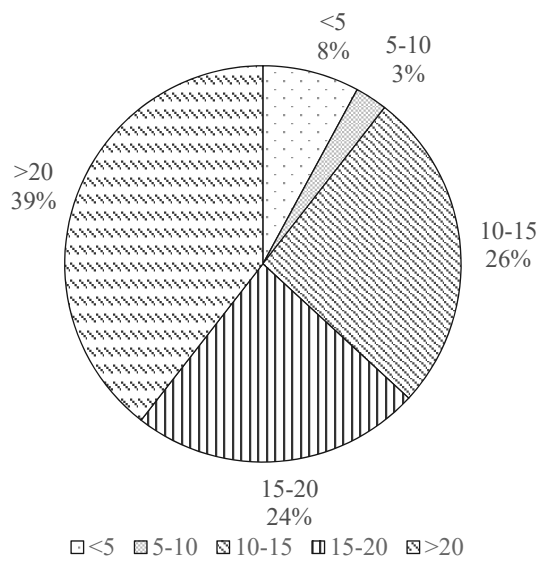

Gráfico 2: ¿Cuántos años hace que se dedica profesionalmente a la economía/a la enseñanza universitaria?

Por su parte, el número total de docentes universitarios, formadores de traductores en este ámbito de especialidad, asciende a 56 (18 hombres y 38 mujeres). La mayor parte de ellos (39) trabaja en España; el resto, en Bélgica (1), Brasil (1), Canadá (1), Francia (2), Irlanda (2), Italia (1), Letonia (1),

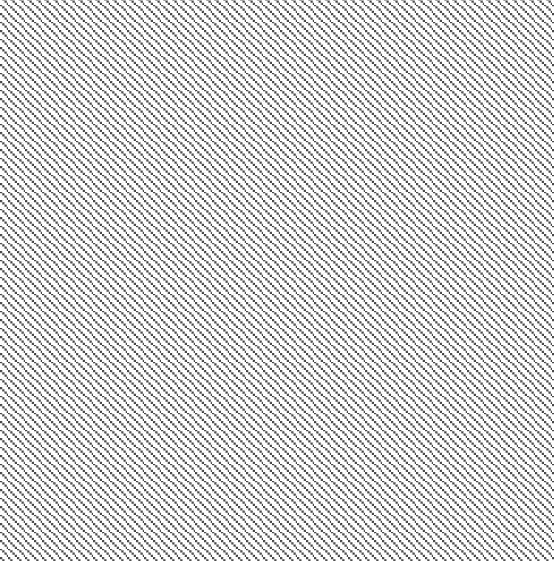

Gráfico 3: Edades de formadores de traductores 
México (1), Polonia (2), Portugal (2), Reino Unido (2) y Suiza (1). Respecto de su edad, la mayor parte de ellos (36\%) tiene entre 30 y 40 años de edad; un $30 \%$ tiene entre 40 y 50 años, y un 23\%, entre 50 y 60 . El gráfico 3 muestra dicha distribución.

Si nos preguntamos en relación con los años de antigüedad en la enseñanza universitaria, así como a los años que han ejercido como traductores profesionales, podemos representar su distribución del siguiente modo:

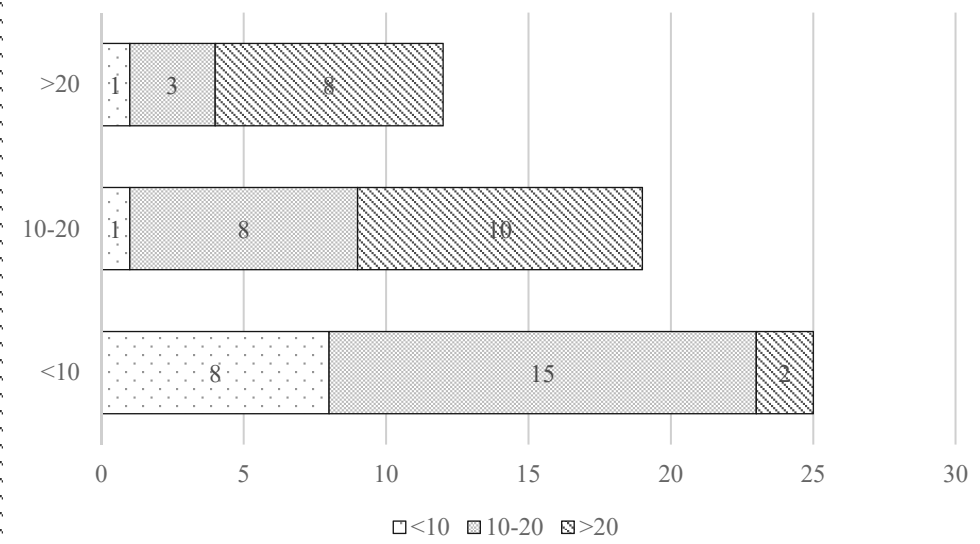

Gráfico 4: Distribución de años en la enseñanza de la traducción y experiencia profesional en traducción

El eje vertical se corresponde con los años que los encuestados llevan en la enseñanza universitaria. El eje horizontal, con los años que han ejercido como traductores. En este gráfico observamos que el grupo más numeroso (25) lleva menos de 10 años en la universidad, si bien la mayor parte (15) tiene entre 10 y 20 años de experiencia profesional. El segundo grupo más numeroso en relación con la enseñanza universitaria es de 19, casi todos ellos con una experiencia profesional de entre 10 y 20 años (8) o más de 20 años (10). Algo similar ocurre con el grupo que cuenta con una experiencia en materia universitaria de más de 20 años (12).

Se trata de formadores que, en su mayoría, tienen estudios de máster (14) y doctorado (39), y que enseñan en grado y máster. Por su parte, el gráfico 5 distribuye los encuestados según el número total de horas que dedican a la traducción económica, comercial y financiera en su programa, así como el nivel en el que imparten clases. El eje vertical se corresponde con el número de horas, distribuidas por franjas de menos de 30 horas, entre 30 y 60 horas, 
entre 60 y 90 horas, y más de 90 horas. El eje horizontal muestra la distribución del número de encuestados según el nivel en el que imparten:

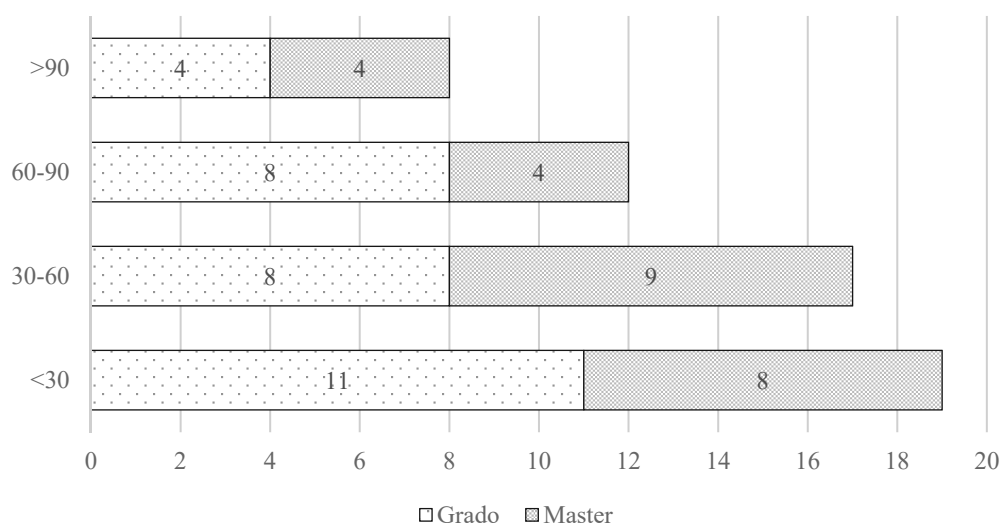

Gráfico 5: ¿En qué nivel enseña traducción económica, comercial o financiera? / ¿Cuál es el número total de horas que dedica a la traducción económica, comercial y financiera en su programa?

\subsubsection{Resultados en torno a la pregunta de materias}

Tanto los profesionales como los formadores respondieron a una pregunta en la que se les proponían diferentes materias o áreas que debían relacionar con la economía, el comercio o las finanzas. La pregunta les permitía relacionarlas con más de un campo. A los formadores de traductores se les preguntó igualmente si incluían alguna de estas áreas en sus clases.

Las tablas 1, 2 y 3 contienen las diecinueve materias que más atribuyeron los profesionales y los formadores a los campos de la economía, el comercio y las finanzas. Cada columna está ordenada de mayor a menor. Cada área viene acompañada del número de encuestados (entre paréntesis) que la marcaron en el cuestionario.

\begin{tabular}{|l|l|}
\hline PROFESIONALES & FORMADORES \\
\hline Macroeconomía (38) & Macroeconomía (51) \\
\hline Socioeconomía (37) & Socioeconomía (50) \\
\hline Mercado de trabajo (36) & Mercado de trabajo (47) \\
\hline Estadística y estudios de mercado (33) & Microeconomía (42) \\
\hline Deuda pública (32) & Deuda pública (41) \\
\hline
\end{tabular}




\begin{tabular}{|l|l|}
\hline Microeconomía (32) & Estadística y estudios de mercado (39) \\
\hline Fiscalidad internacional (25) & Fiscalidad internacional (36) \\
\hline Comercio exterior (24) & Gobierno corporativo (34) \\
\hline Mercados financieros (24) & Administración de empresas (33) \\
\hline Gobierno corporativo (21) & Informes fiscales (32) \\
\hline Informes fiscales (19) & Mercados financieros (31) \\
\hline Administración de empresas (18) & Documentos societarios (27) \\
\hline Contratos de alta dirección (18) & Contabilidad de gestión o directiva (27) \\
\hline Desarrollo de negocio (18) & Seguros (27) \\
\hline Financiación (18) & Logística (27) \\
\hline Auditoría y consultoría (17) & Contratos de alta dirección (26) \\
\hline Bolsa (17) & Recursos Humanos (26) \\
\hline $\begin{array}{l}\text { Información para accionistas e inversores } \\
\text { (17) }\end{array}$ & $\begin{array}{l}\text { Información para accionistas e inversores } \\
(25)\end{array}$ \\
\hline Documentos societarios (16) & Análisis de riesgo (25) \\
\hline
\end{tabular}

Tabla 1: Campo de la economía

Según los resultados mostrados en la tabla 1, en el campo de la economía, coinciden 14 de los 19 ítems, aunque el orden de clasificación difiere. Este fuerte consenso entre ambos grupos queda reflejado especialmente en los siete primeros ítems, que aluden a las mismas áreas (cinco de los cuales tienen el mismo ranking). Dicho consenso podría indicar que lo que los economistas consideran elementos centrales en el sector económico queda cubierto en el aula. Por su parte, las diferencias son reveladoras: mientras que los economistas consideran importantes la auditoría y consultoría, el desarrollo de negocios, la financiación, el comercio exterior y la bolsa, los formadores no solo los omiten, sino que incluyen otras áreas, como los recursos humanos, los seguros, la logística, la contabilidad de gestión o directiva, y el análisis de riesgo. Por último, un ítem en concreto, el comercio exterior, parece ser la diferencia más obvia, aunque en la tabla 2 se muestra el consenso en la asignación de comercio exterior al sector comercial.

\begin{tabular}{|l|l|}
\hline PROFESIONALES & FORMADORES \\
\hline Marketing (34) & Comercio exterior (45) \\
\hline Comercio exterior (32) & Marketing (44) \\
\hline Logística (32) & Negocio al por menor (42) \\
\hline
\end{tabular}




\begin{tabular}{|l|l|}
\hline Negocio al por menor (30) & $\begin{array}{l}\text { Presupuestos, órdenes de compras, notas } \\
\text { de abono (41) }\end{array}$ \\
\hline $\begin{array}{l}\text { Presupuestos, órdenes de compras, notas } \\
\text { de abono (26) }\end{array}$ & Logística (38) \\
\hline Protocolo (24) & Recursos Humanos (38) \\
\hline Banca comercial (21) & Desarrollo de negocio (37) \\
\hline Desarrollo de negocio (21) & Contratos mercantiles (36) \\
\hline Administración de empresas (19) & Instrumentos negociables (29) \\
\hline Contratos mercantiles (19) & Banca comercial (28) \\
\hline Instrumentos negociables (17) & Estadística y estudios de mercado (26) \\
\hline Recursos Humanos (17) & Administración de empresas (25) \\
\hline Seguros (16) & Seguros (25) \\
\hline $\begin{array}{l}\text { Documentos financieros del ámbito } \\
\text { internacional (15) }\end{array}$ & Protocolo (24) \\
\hline Documentos societarios (14) & Documentos societarios (24) \\
\hline Estadística y estudios de mercado (14) & Gobierno corporativo (23) \\
\hline Gobierno corporativo (14) & Contratos de alta dirección (23) \\
\hline Contratos de alta dirección (13) & Mercado de trabajo (21) \\
\hline Microeconomía (11) & $\begin{array}{l}\text { Contratos de compraventa de acciones } \\
\text { (19) }\end{array}$ \\
\hline & \\
\hline
\end{tabular}

Tabla 2: Campo del comercio

En el sector comercial, de nuevo, también hay consenso entre ambos grupos, en este caso incluso más importante que en el campo anterior, con 17 ítems coincidentes de 19. Los cinco primeros ítems son idénticos, aunque difiere el orden (dado el número relativamente pequeño, este dato puede no ser significativo). Los profesionales, además, incluyeron documentos financieros del ámbito internacional, y microeconomía, mientras que los formadores de traductores incluyeron el mercado de trabajo y contratos de compraventa de acciones. En este sentido, los resultados parecen apuntar que los formadores de traductores cubren bien este campo. Dado que el comercio exterior aparece tanto en la tabla 2 como en la tabla 1, relativa al campo de la economía, sería muy interesante y útil que los formadores, a la hora de diseñar sus cursos, se plantearan dónde exactamente resulta más conveniente incluir esta materia. 


\begin{tabular}{|l|l|}
\hline PROFESIONALES & FORMADORES \\
\hline Instrumentos financieros (38) & Banca de inversión (51) \\
\hline Banca de inversión (37) & Instrumentos financieros (48) \\
\hline Project finance (37) & Bolsa (47) \\
\hline Bolsa (36) & $\begin{array}{l}\text { Documentos financieros del ámbito } \\
\text { internacional (47) }\end{array}$ \\
\hline Financiación (36) & Finanzas corporativas (46) \\
\hline Contratos financieros (35) & Contratos financieros (45) \\
\hline Finanzas corporativas (35) & Certificados de acciones (45) \\
\hline Análisis de riesgo (34) & Financiación (44) \\
\hline Operaciones corporativas (33) & Análisis de riesgo (44) \\
\hline $\begin{array}{l}\text { Información para accionistas e inversores } \\
\text { (32) }\end{array}$ & Mercados financieros (43) \\
\hline Auditoría y consultoría (31) & Contratos del ámbito bancario (43) \\
\hline Mercados financieros (31) & Project finance (42) \\
\hline Certificados de acciones (30) & Planes de opciones sobre acciones (42) \\
\hline Planes de opciones sobre acciones (30) & Auditoría y consultoría (39) \\
\hline Contabilidad (29) & $\begin{array}{l}\text { Información para accionistas e inversores } \\
\text { (37) }\end{array}$ \\
\hline Contabilidad de gestión o directiva (29) & Contabilidad (36) \\
\hline $\begin{array}{l}\text { Contratos de compraventa de acciones } \\
\text { (29) }\end{array}$ & $\begin{array}{l}\text { Contratos de compraventa de acciones } \\
\text { (33) }\end{array}$ \\
\hline Contratos del ámbito bancario (29) & Banca comercial (33) \\
\hline $\begin{array}{l}\text { Documentos financieros del ámbito } \\
\text { internacional (28) }\end{array}$ & Instrumentos negociables (33) \\
\hline
\end{tabular}

Tabla 3: Campo de las finanzas

Los datos sobre el campo de las finanzas son similares a los del comercial, ya que 17 de los 19 ítems coinciden, lo que vuelve a indicar un amplio consenso entre los profesionales y los formadores (y posiblemente una estrecha relación entre los formadores de traductores y el sector al que sirven). Por el lado de los profesionales, las operaciones corporativas se sitúan en medio de la tabla, y la contabilidad de gestión o directiva, más abajo. En cambio, por el lado de los formadores, la banca comercial y los instrumentos negociables se sitúan en el ranking más bajo. Mientras que la contabilidad de gestión o directiva podría incluirse dentro de la materia de contabilidad general, los formadores 
podrían considerar incluir la compleja materia de operaciones corporativas dentro de sus planes docentes.

\subsubsection{Resultados en torno a la formación y la investigación}

En este apartado presentamos los resultados que tienen que ver con varios conceptos relativos a la formación en el ámbito de la traducción económica, así como a la investigación en este campo. En concreto, nos interesaba conocer qué entienden otros docentes del ámbito universitario por teoría dentro de la traducción económica, además de saber su parecer sobre la necesidad de impartir conocimientos especializados en economía, comercio y finanzas en las asignaturas de traducción o de adquirir estos conocimientos a la hora de traducir. Asimismo, nos preocupamos por averiguar cuáles son las líneas de investigación que, según los encuestados, merecen mayor atención.

La tabla 4 contiene las materias, según los resultados, suelen enseñarse con más frecuencia en grado y en máster. ${ }^{4}$ Cada columna está ordenada de mayor a menor. Cada materia viene acompañada del número de encuestados que la marcaron en el cuestionario:

\begin{tabular}{|l|l|}
\hline GRADO & MÁSTER \\
\hline Comercio exterior (18) & Contabilidad (14) \\
\hline Documentos societarios (18) & $\begin{array}{l}\text { Información para accionistas e inversores } \\
(13)\end{array}$ \\
\hline Bolsa (15) & Comercio exterior (12) \\
\hline Contabilidad (15) & Documentos societarios (12) \\
\hline Contratos mercantiles (15) & Mercados financieros (12) \\
\hline Macroeconomía (15) & Contratos mercantiles (11) \\
\hline Comercio (14) & Banca comercial (11) \\
\hline $\begin{array}{l}\text { Información para accionistas e inversores } \\
(14)\end{array}$ & Bolsa (10) \\
\hline Mercados financieros (14) & Comercio (9) \\
\hline Seguros (14) & Mercado de trabajo (9) \\
\hline Mercado de trabajo (13) & Administración de empresas (9) \\
\hline
\end{tabular}

4. Téngase en cuenta que estos resultados no se aplican a los Estados Unidos, donde el estudio de lenguas extranjeras no está muy desarrollado en la enseñanza secundaria, lo que significa que hay muy pocos grados en traducción y que los que existen se centran principalmente en el desarrollo de competencias lingüísticas y no tanto en habilidades relacionadas con la traducción especializada. 


\begin{tabular}{|l|l|}
\hline Administración de empresas (12) & $\begin{array}{l}\text { Documentos financieros del ámbito } \\
\text { internacional (9) }\end{array}$ \\
\hline Banca comercial (12) & Marketing (9) \\
\hline $\begin{array}{l}\text { Documentos financieros del ámbito } \\
\text { internacional (11) }\end{array}$ & Instrumentos financieros (8) \\
\hline Microeconomía (11) & Instrumentos negociables (8) \\
\hline Contratos del ámbito bancario (10) & Finanzas corporativas (8) \\
\hline Deuda pública (10) & Gobierno corporativo (8) \\
\hline Instrumentos financieros (9) & Seguros (7) \\
\hline Instrumentos negociables (9) & Deuda pública (7) \\
\hline Auditoría y consultoría (8) & Contratos financieros (7) \\
\hline Socioeconomía (8) & Financiación (7) \\
\hline
\end{tabular}

Tabla 4: ¿Qué materias o áreas enseñan los formadores?

Los dos niveles coinciden en 16 de las 21 áreas temáticas, lo cual no es de extrañar, pues los temas que se enseñan de manera introductoria en los estudios de grado pueden ampliarse en los de máster. Según estos datos, los temas de los másteres también incluyen las finanzas corporativas, el gobierno corporativo, los contratos financieros, la financiación y el márketing, algo que no sucede en el grado. Todos estos elementos aparecen en la mitad inferior de la lista. El grado añade auditoría y consultoría, contratos del ámbito bancario, macroeconomía, microeconomía, y socioeconomía. Los últimos tres ítems pueden ser considerados más teóricos que el resto de ítems. De nuevo, las diferencias se encuentran en la mitad inferior de la tabla, a excepción de la macroeconomía, lo que indica que las áreas temáticas centrales (situadas en la mitad superior de la lista) coinciden en los grados y másteres de traducción.

El gráfico 6, por su parte, muestra los resultados en torno a la pregunta sobre el término teoría.

Según los resultados, el término teoría muestra una gran inestabilidad conceptual, pues, entre otras cosas, puede entenderse no solo como los conocimientos sobre la materia para la que se traduce (41 encuestados seleccionaron esta opción), sino también como conocimientos generales en el ámbito de la economía, comercio y finanzas (43 encuestados seleccionaron esta opción). Esta ambivalencia podría a interpretarse como diferentes maneras de aproximarse a los conocimientos teóricos en el ámbito de la formación de traductores económicos: ¿debería presentarse primero el encargo y, a partir de él, aproximarse a los conocimientos teóricos que se desprenden de él? , ¿o cabría 


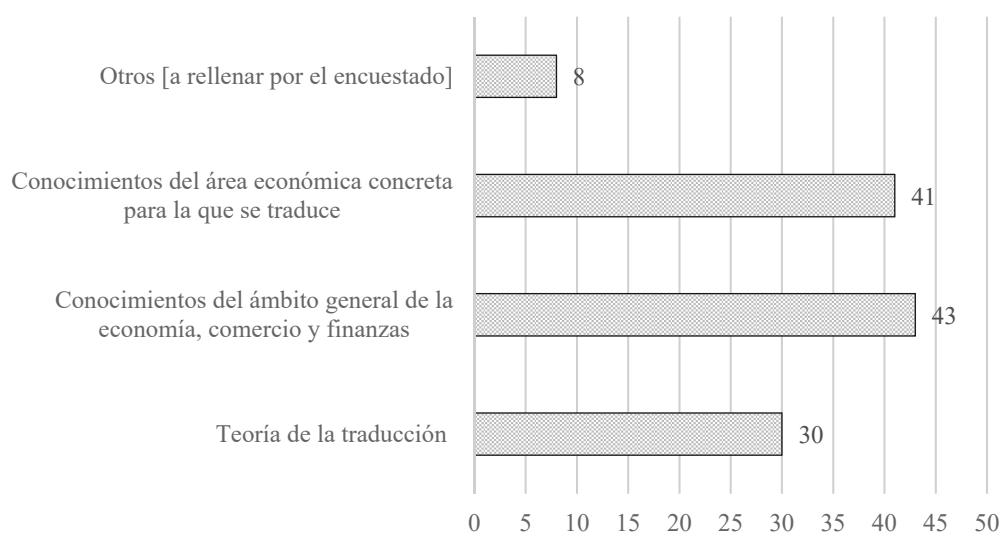

Gráfico 6: En el marco de la traducción económica, comercial o financiera, ¿qué es para usted la teoría?

dotar al traductor en formación de una serie de conocimientos de base antes de afrontar la traducción? Los comentarios de los encuestados a la hora de describir el término teoría también apuntan a que el concepto teoría puede interpretarse igualmente como teoría de la traducción, lo que puede llevar a pensar en la necesidad de hacer hincapié en determinados aspectos traductológicos que puedan resultar de interés para la traducción económica, comercial y financiera, tales como los conocimientos generales sobre la práctica de la traducción especializada o sobre estrategias de traducción.

Respecto de la pregunta relacionada con la importancia de la teoría (ien qué medida considera que la teoría es esencial para la práctica de la traducción económica, comercial y financiera?), los encuestados proporcionaron 55 respuestas libres, cuyo análisis revela dos ejes bien distintos. El primero se refiere a la importancia de la teoría según los encuestados, con respuestas que pueden dividirse en cuatro grupos: muy positivas, comentarios generales, conocimientos básicos y negativas. El segundo eje se refiere a cómo debe entenderse el término teoría, pues la pregunta parecía ser ambigua. Algunos encuestados relacionaron esta pregunta con la teoría de la traducción, mientras que otros lo hicieron con las áreas y materias en cuestión. A continuación, presentamos cada grupo según la importancia del eje, desglosado por los aspectos teóricos.

\section{Respuestas muy positivas}

La mayor parte de las respuestas $(37,67 \%)$ fueron positivas e indicaban que la teoría es "esencial", "importante", "indispensable" u otros calificativos 
similares, aunque dos indicaron alguna salvedad al respecto. En estas respuestas los encuestados parecen estar de acuerdo en que una adecuada base teórica resulta fundamental para hacer una buena traducción. De estos 37 encuestados, 20 (36\%) no indicaron el tipo de teoría al que se referían.

Siete encuestados que consideran importante la teoría se refirieron específicamente tanto a la teoría de traducción como a la teoría entendida como conocimientos de áreas o materias propias del ámbito de especialidad. En relación con la teoría de la traducción algunas respuestas apuntan a aspectos como teoría de la traducción especializada, conocimientos sobre estrategias de traducción y procesos documentales o la conciencia de la importancia del enfoque funcionalista de la traducción, entre otros. Asimismo, los encuestados conciben la teoría como teoría sobre la economía, negocios y finanzas. Uno de los encuestados apunta la transversalidad como indispensable para la traducción en este ámbito de especialidad, esto es, la existencia de asignaturas de economía aplicables a los estudiantes de traducción en esta materia.

Uno de los encuestados no considera que la teoría sea tan necesaria al principio como las habilidades instrumentales. Este docente en la primera fase de formación considera más importante la aplicación de competencias instrumentales para la resolución de tareas de traducción concretas.

Los comentarios del siguiente grupo, que consta de ocho respuestas positivas, consideran específicamente que el conocimiento de la materia es un aspecto importante. Las respuestas apuntan al menos a la necesidad de adquirir determinados conocimientos básicos o indispensables para la traducción en el ámbito de especialidad. Asimismo, algunos docentes resaltan la necesidad de dominar la terminología del área del saber y de dotar al alumno de las herramientas y recursos necesarios para adquirir esos conocimientos y terminología. Una de las respuestas incluso rechaza específicamente la teoría de la traducción ("La traductología aporta poco a la práctica de la traducción"). Uno de los encuestados apunta la necesidad en este campo de especialidad de tener ciertos conocimientos sobre la traducción de textos jurídicos, incluyendo las referencias a otros sistemas jurídicos y judiciales

\section{Comentarios generales}

Seis respuestas (10\%) fueron de carácter general, es decir, no abordaron el nivel de importancia de la teoría. Tres de ellas ni siquiera tenían que ver con la teoría. Algunas respuestas apuntan aspectos como la importancia de sistematizar los problemas de traducción que puedan surgir y sus posibles soluciones, la reflexión sobre el destinatario del texto origen, estrategias de traducción, metodologías de traducción basadas en las características del discurso, etc. 


\section{Conocimientos básicos}

Ocho encuestados (14\%) afirmaron que el conocimiento básico es necesario. Uno no hizo ningún comentario sobre teoría; tres aludieron al conocimiento de la materia, dos mencionaron la teoría de la traducción, y dos hablaron sobre conocimientos básicos en ambas áreas.

\section{Comentarios negativos}

Cuatro encuestados (7\%) indicaron que la teoría no es importante. Dos simplemente declararon que "no es necesaria". Los otros dos prefirieron hablar de metodología y conocimiento, respectivamente.

Dejando de lado la pregunta referida a la importancia de la teoría y tomando en consideración la relacionada con la necesidad de impartir conocimientos especializados en materias de traducción, la gran mayoría de los formadores (93\%) sostiene que sí.

Asimismo, entre los aspectos didácticos que consideran más importantes, los formadores prefieren los terminológicos y textuales, muy por delante de los comunicativos $\mathrm{u}$ otros aspectos: ${ }^{5}$

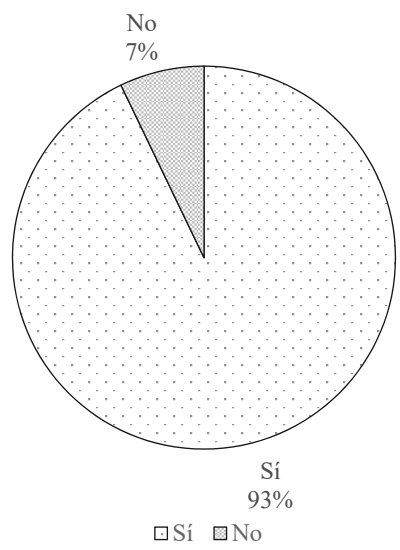

Gráfico 7: ¿Considera necesario la impartición de conocimientos económicos, comerciales y financieros en materias de traducción económica, comercial y financiera?

5. Los comentarios correspondientes a la categoría otros fueron los siguientes: fraseología en gran medida; especializados del campo; conocimientos del área= conceptos; culturales, los documentos dependen de la cultura; temática; conceptos económicos, comerciales o financieros; conceptuales y fraseológicos; procesos de traducción especializada; documentales; conceptos clave del ámbito de especialidad; discursivos y genéricos; también salidas laborales, aspectos del comercio; ver arriba; conceptuales; fraseología, mayor contexto. 


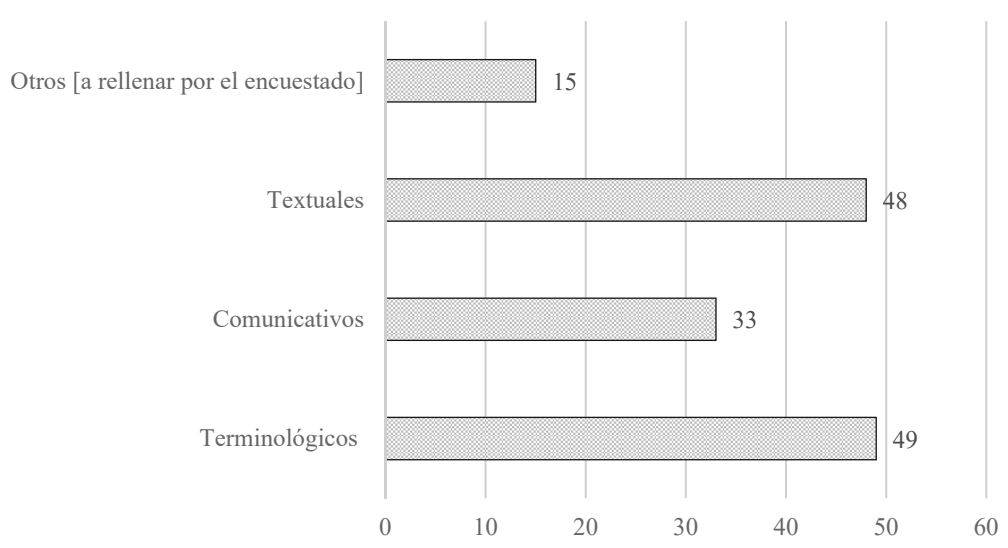

Gráfico 8: ¿Qué aspectos considera que son más importantes a la hora de enseñar la traducción económica, comercial y financiera?

\subsection{Análisis de resultados}

En este trabajo basado en encuestas hemos tratado de dar respuesta a algunos debates abiertos en el panorama investigador, como son el de la delimitación del concepto traducción económica y las materias que, entre otros aspectos, engloba dicho concepto, así como otras cuestiones, como la importancia de la teoría en este ámbito de especialidad o las materias que los formadoresinvestigadores consideran más interesantes como objeto de investigación.

En primer lugar, si analizamos las respuestas de los formadores en traducción y de los especialistas relativas a las distintas materias presentadas en la encuesta y que pueden enmarcarse bajo las etiquetas de economía, comercio y finanzas, podemos concluir que, si bien hay determinadas materias, como la macroeconomía o la socioeconomía, en las que existe un claro consenso a la hora de incluirlas en determinadas etiquetas (por ejemplo, casi la totalidad de los formadores y de los especialistas las incluyen en la etiqueta economía), el marketing y el comercio exterior (la mayoría de los especialistas y de los formadores las incluyen bajo la etiqueta comercio), instrumentos financieros, banca de inversión y bolsa (casi la totalidad de los formadores y especialistas también las incluyen en la etiqueta finanzas); hay otras materias que no se presentan de manera irrefutable bajo una u otra etiqueta. Así, por ejemplo, observamos que la materia bolsa se incluye tanto bajo la etiqueta economía como finanzas. Lo mismo sucede con el análisis de riesgo, materia que se presenta tanto en el ámbito de la economía como en el de las finanzas. Estos resultados ponen de manifiesto uno de los comentarios más recurrentes que 
los especialistas y los formadores nos transmitieron una vez cumplimentada la encuesta, esto es, la imposibilidad de asignar una u otra materia a un área de conocimiento determinada. A la luz de los resultados y con la excepción de determinadas materias, hay múltiples materias que se adscriben a más de una etiqueta, por lo que a menudo resulta imposible que todas las subespecialidades se enmarquen en una única área del saber.

Por otro lado, podemos apreciar un amplio consenso entre profesionales y formadores relativo a las áreas temáticas incluidas en cada categoría (14 de las 19 principales categorías en el campo de la economía, 17 de 19 en el del comercio, y 17 de 19 en el campo de las finanzas). Esto podría indicar dos aspectos positivos: primero, que lo que los traductores en formación están aprendiendo en clase de traducción especializada cubre en gran medida lo que los profesionales del campo consideran necesario, y, segundo, que los formadores entienden considerablemente lo que los campos especializados requieren. Ello, a su vez, podría indicar que los propios formadores de traductores son verdaderos expertos en las materias de cada campo o que al menos tienen bastante contacto con el campo.

Existen otras materias en las que se puede comprobar una divergencia importante entre la visión que tienen los especialistas y la que tienen los formadores, por ejemplo, bajo la etiqueta de economía el comercio exterior se posiciona como la octava materia más elegida entre los especialistas y ni siquiera se contempla entre las elecciones de los formadores. Los formadores, por su parte, incluyen el comercio exterior bajo la etiqueta comercio. Lo mismo sucede con las materias desarrollo de negocio, financiación o auditoría y consultoría que son valoradas por un gran número de especialistas bajo la etiqueta de economía y no aparecen en absoluto contempladas entre los formadores como economía. Por ejemplo, los formadores incluyen la materia desarrollo de negocio en el campo de comercio y la financiación, en el campo de finanzas. Desde el punto de vista curricular, probablemente no es tan importante el hecho de conocer con exactitud el lugar en el que debe enmarcarse un tema o área como el hecho de que este sea incluido en el curso o asignatura. Ello puede significar que los formadores de traductores deberían reorganizar sus planes docentes de algún modo; por ejemplo, puede ser útil añadir la categoría de comercio exterior en una asignatura de traducción económica, pero quizá con un mayor énfasis en los aspectos económicos sobre los aspectos comerciales.

Respecto de las respuestas de los formadores sobre las materias enseñadas en grado y en máster, podemos concluir que, salvo excepciones (por ejemplo 
la macroeconomía es la sexta materia enseñada en grado, pero no se enseña en máster), las materias enseñadas en grado no difieren mucho de las materias enseñadas en máster y suelen ocupar más o menos el mismo orden de frecuencia, esto es, el número de encuestados que han elegido una u otra materia no difiere significativamente. Por ejemplo, la materia más enseñada en grado es el comercio exterior y la más enseñada en máster es la contabilidad. Por su parte, la contabilidad es la tercera materia más enseñada en grado y el comercio exterior, la tercera más enseñada en máster. Asimismo, por ejemplo, los documentos societarios ocupan el segundo puesto en grado y el cuarto en máster y los contratos mercantiles ocupan el quinto puesto en grado y el sexto en máster.

En nuestro estudio también hemos preguntado a los formadores cómo conciben el término teoría. De los resultados obtenidos se desprende la conclusión de que la teoría se concibe casi en igual medida tanto como teoría del área temática concreta objeto de traducción como teoría general del ámbito de la economía, comercio y finanzas. Asimismo, tal y como se deduce de los comentarios de una mayoría de los encuestados, la teoría también puede interpretarse como teoría de la traducción, esto es, conocimientos teóricos relacionados con la traducción especializada y otros conocimientos relacionados con algunas competencias traductoras necesarias en el ámbito de especialidad como la documental, terminológica o fraseológica. Para el 93\% de los formadores encuestados es necesario impartir conocimientos económicos, financieros y comerciales en este ámbito de especialidad. Es indudable que, a juzgar por los comentarios de los encuestados en esta parte de nuestro estudio, la teoría resulta esencial. En futuros estudios basados en encuestas, podría resultar de utilidad diferenciar en los cuestionarios entre "teoría de la traducción", "teoría de la materia ", y "conocimiento de la materia" con el fin de obtener una idea más clara de la importancia relativa y el énfasis que se debe dar a las dos áreas.

Para los formadores en traducción en esta materia los aspectos más importantes a la hora de enseñar son los terminológicos, seguidos de los textuales y, en menor medida, los aspectos comunicativos.

Uno de los resultados más sorprendentes del presente estudio es la muy diferente visión que tienen los especialistas y los formadores en la materia sobre la necesidad de ser experto para traducir en este ámbito de especialidad (cf. gráfico 9). Un 90\% de los especialistas en la materia considera que es necesario ser experto en este ámbito frente al 20\% de los formadores. Esta visión tan diferente de este asunto nos lleva a plantearnos si los especialistas 
que han cumplimentado la encuesta son conscientes de lo que entendemos los formadores en traducción por experto (el profesional en la materia distinto del traductor con conocimientos en la materia) y de las competencias que se exigen al traductor que exceden la mera competencia lingüística.

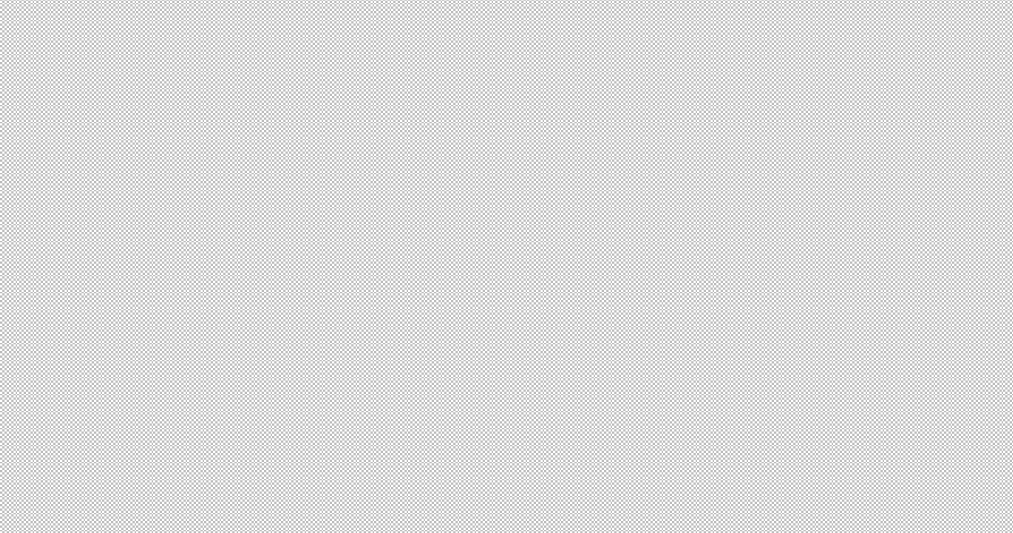

Gráfico 9: ¿Cree que es necesario ser un experto en economía, comercio o finanzas para traducir textos económicos, comerciales o financieros?

Las respuestas de los profesionales pueden estar basadas en una concepción ingenua del proceso de traducción (al fin y al cabo, los traductores no necesitan crear nuevos textos de economía, comercio o finanzas, sino, más bien, transferir el significado ya creado por expertos a un texto meta bien formado, utilizando terminología y fraseología relevante). Esto implica que los traductores tienen unas habilidades o competencias distintas a las de los especialistas profesionales, una pericia reproductiva, comprehensiva y receptiva, que es de naturaleza fundamentalmente diferente a la de cualquier profesional de otro campo.

En cuanto a la investigación en traducción económica, tal como muestra el gráfico 10, los aspectos que los encuestados han señalado como más importantes son la enseñanza de la traducción económica, comercial y financiera, seguidos de los estudios contrastivos de géneros textuales de la economía, comercio y finanzas, del desarrollo de productos terminográficos, estudios cognitivos sobre el proceso de traducción en la materia y por último de estudios sociales relacionados con el perfil del traductor económico, comercial y financiero. 


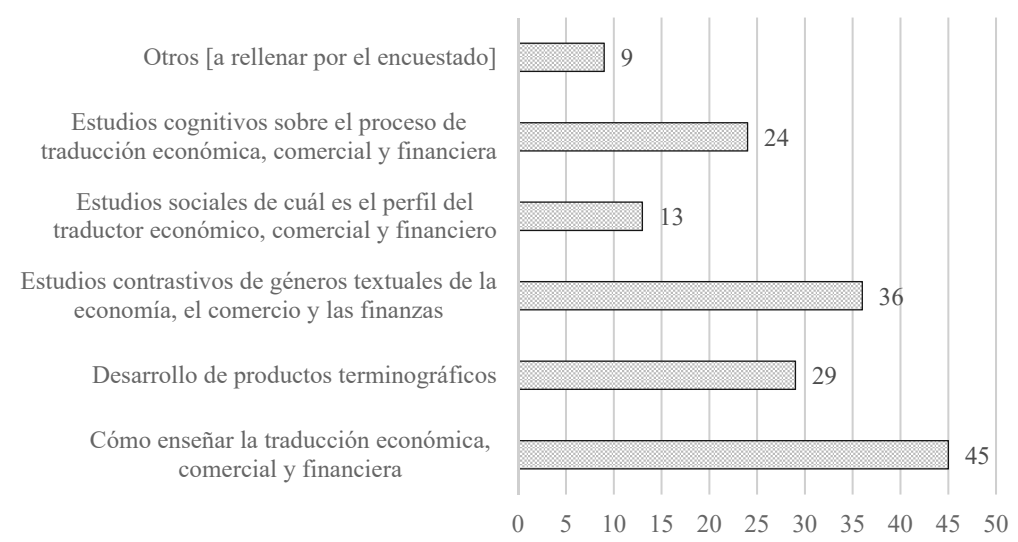

Gráfico 10: ¿En qué aspectos piensa que el investigador en traducción económica, comercial y financiera debería hacer más hincapié? ${ }^{6}$

\section{Conclusiones}

Los resultados de este estudio indican, en esencia, que los temas que los profesionales consideran más importantes son los que, de hecho, imparten los formadores de traductores en su mayor parte. Este resultado positivo significa que (si la encuesta es suficientemente representativa) los formadores de traductores no tienen por qué llevar a cabo una renovación curricular significativa. En algunos casos, quizá sí que pueda ser necesario ajustar algunos parámetros curriculares con el ánimo de que esta idónea selección de temas vaya más en línea con las materias que los profesionales consideran importantes.

Por otro lado, sería interesante (aunque difícil) realizar encuestas dirigidas a las agencias de traducción y a las grandes empresas que solicitan servicios de traducción en estos ámbitos de especialidad con el fin de confirmar si los temas más enseñados en el aula son también los más traducidos en el mercado, pues podría argumentarse que, para un programa de estudios de traducción, son más interesantes los temas "más traducidos" que los más significativos dentro de una determinada especialidad.

6. Los comentarios correspondientes a la categoría otros fueron los siguientes: 1) principios de traducción aplicados a conceptos clave, 2) enseñanza de utilización de recursos, 3) la adquisición del conocimiento experto, 4) estudios descriptivos sobre traducciones profesión, 5) las TIC y el comercio, 6) bases de datos de traducciones comentadas, 7) conocimientos conceptuales del campo, 8) no sé, 9) problemas y soluciones de traducción. 
Por último, puede ser necesario proporcionar "educación del cliente" para profesionales en el campo en cuanto a las diferencias entre los expertos en un campo profesional determinado y la experiencia de un "traductor económico, comercial y financiero experto".

\section{Referencias bibliográficas}

BoCQUET, Claude. (1993) Traduire les textes économiques, financiers et bancaires allemands aux différents niveaux du discours. Prilly, Laussane: CB Service.

Durban, Chris. (2005) "La traduction financière. Tendances pour l'avenir." In: Gouadec, Daniel (ed.) 2005. Traduction, Terminologie, Rédaction. Actes des universités d'été et d'automne et du colloque international Traduction spécialisée chemins parcourus et autoroutes à venir traduire pour le web. Paris: La maison du dictionnaire, pp. 63-70.

Gallego-HernándeZ, Daniel. (2012) Traducción económica y corpus. Del concepto a la concordancia. Alicante: Universidad de Alicante.

Gallego-Hernández, Daniel (ed.) (2014) Traducción económica: entre profesión, formación y recursos documentales. Soria: Diputación Provincial.

GOUADEC, Daniel. (2007) Translation as a Profession. Amsterdam: John Benjamins.

GUÉVEL, Zélie. (1990) "Traduction et développement de la terminologie française dans le domaine des affaires." Meta 35:1, pp. 154-161.

GUIDÈRE, Mathieu. (2008) La communication multilingue. Traduction commerciale et institutionnelle. Bruxelles: De Boeck.

HERAS DíEZ, Florentino. (2005) Materiales para la traducción económico-financiera francés-español. Alicante: Club Universitario.

Lahlali, El Mustapha \& Wafa Abu Hatab. (2014) Advanced English-Arabic Translation: A Practical Guide. Edinburgh: Edinburgh University Press.

LIE, Raymond S. C. (1995) "Commercial Translation." In: Chan, Sin-wai \& David E. Pollard (eds.) 1995. Encyclopedia of Translation. Hong Kong: Chinese University Press, pp. 95-109.

Llombart Rosa, Vicent. (2009) "Traducciones de economía." In: Lafarga Maduell, Francisco (ed.) 2009. Diccionario histórico de la traducción en España. Madrid: Gredos, pp. 325-334.

MAYORAL ASENSIO, Roberto. (2007) "La traducción comercial." In: Fuertes Olivera, Pedro A. (ed.) 2007. Problemas lingüísticos en la traducción especializada. Valladolid: Universidad, pp. 33-47.

OlOHAN, Maeve. (2009) "Commerical Translation." In Baker, Mona \& Gabriela Saldanha (eds.) 2009. Routledge Encyclopedia of Translation Studies, $2^{\text {nd }}$ edition. New York: Routledge, pp. 40-42.

Rochard, Michel. (2005) "La traduction financière à la croisée des chemins." Traduire 204, pp. 7-16. 
RomÁn MíngueZ, Verónica. (2015) "La traducción de textos económicos: principales características y dificultades.” In: Penas Ibáñez, Azucena (ed.) 2015. La traducción: nuevos planteamientos teórico-metodológicos. Madrid: Síntesis, pp. 139-155.

Svendsen, Lisbet Pals. (2001) "Economic Translation. How to Put Theory into Practice." In: Chabás, José; Madeleine Cases \& Rolf Gaser (eds.) 2001. Proceedings. First International Conference on Specialized Translation. Barcelona: Universitat Pompeu Fabra, pp. 39-41.

Visauta Vinacua, Bienvenido. (1989) Técnicas de investigación social. I Recogida de datos. Barcelona: PPU.

\section{NOTAS BIOGRÁFICAS}

DANiEl GALlEGO-HeRnÁNDEZ es doctor en Traducción e Interpretación y actualmente imparte clases de traducción económica y terminología en el Departamento de Traducción e Interpretación de la Universidad de Alicante. Desde que defendió su tesis, Traducción económica y textos paralelos en internet, ha centrado su docencia e investigación en la traducción económica, comercial y financiera en su relación con la Lingüística de corpus, la formación y la terminología. Ha publicado el libro Traducción económica y corpus: del concepto a la concordancia.

VERÓNICA ROMÁn MíngueZ es licenciada en Derecho por la Universidad de Valladolid y licenciada en Traducción e Interpretación por la Universidad Complutense de Madrid. Asimismo, es traductora-intérprete jurada de lengua inglesa por el Ministerio de Asuntos Exteriores y Cooperación de España y doctora en Traducción e Interpretación por la Universidad de Málaga. Se dedica profesionalmente a la traducción desde 1995. Desde 2006 desarrolla su labor docente en la Universidad Autónoma de Madrid. Sus líneas de investigación se centran en la enseñanza-aprendizaje de la traducción económicofinanciera y jurídica (inglés-español), así como en el mercado profesional de la traducción.

GeOfFrey S. Koby, doctor en lingüística de alemán, es Profesor Titular de alemán y traducción en el Instituto de Lingüística Aplicada de la Universidad Estatal de Kent (Ohio, EEUU). Su investigación se centra en la evaluación y pedagogía de la traducción. En la actualidad, su trabajo está encaminado a desarrollar una base de datos que almacena un corpus de exámenes de certificación de la Asociación Americana de Traductores (ATA, American Translators Association), así como investigar los aspectos cuantitativos y cualitativos de 
estos exámenes. Actualmente es miembro del Consejo de Administración de la ATA. Entre sus publicaciones recientes se encuentra "The ATA Flowchart and Framework as a Differentiated Error-Marking Scale in Translation Teaching" (2015). 